\title{
Can Graphene Lead to Breakthrough in Boiling Heat Transfer?
}

\author{
Hyung Hee Cho*, Geehong Choi, and Dong II Shim \\ Department of Mechanical Engineering, Yonsei University, 50 Yonsei-ro, Seodaemun-gu, Seoul 120-749, Korea
}

*Corresponding author: Hyung Hee Cho, Department of Mechanical Engineering, Yonsei University, 50 Yonsei-ro, Seodaemun-gu, Seoul 120-749, Korea, Tel: +82-2-2123-2828; Fax: +82-2-312-2159; E-mail: hhcho@yonsei.ac.kr

Rec date: Mar 10, 2014, Acc date: Mar 13, 2014, Pub date: Mar 18, 2014

Copyright: (C) $2014 \mathrm{Cho} \mathrm{HH}$, et al. This is an open-access article distributed under the terms of the Creative Commons Attribution License, which permits unrestricted use, distribution, and reproduction in any medium, provided the original author and source are credited.

\section{Introduction}

It is within bounds to say that Graphene is at the cutting edge of material science and various engineering fields. Representative research and applications can be readily found in composite material, semiconductor, display, and energy for its intrinsic and potential merits involving electrical, mechanical and physico-chemical characteristics. The powerful merits of Graphene have been adapted in lots of application fields through the development of reliable and feasible synthesis technology. Especially for heat transfer applications, we can also highlight its attractiveness on interfacial functionalization with extremely high thermal conductivity [1], which is one order of magnitude greater than even common conductive materials such as copper or aluminum (with the order of hundreds of $\mathrm{W} / \mathrm{m} \cdot \mathrm{K}$ ).

Based on the outstanding properties, Graphene has been applied to thermal science and technology including boiling heat transfer which is used in heat-exchanger, boiler, cooling for electric devices [2]. On boiling heat transfer, surface morphology and wettability characteristics have been demonstrated as principal determinants $[3,4]$. Effective nucleation sites can be favorable to catalyze heterogeneous vaporization on an interfacial surface [5]. Refreshment of coolant on the surface can also be manipulated against dry-out of the surface during the phase-changing phenomena [6]. Herewith, Graphene can be an influential candidate to suggest a breakthrough for the enhancement of cooling performances. Even though the physics on thermophoretic behavior of Graphene is not clear, surface functionalization by Graphene has been demonstrated to manipulate surface roughness and wettability, and to improve reliability and performance of heat dissipation.

Figure $1[7,8]$ presents typical boiling performance curves and describes the possibility of Graphene for a potential breakthrough in performance improvement of boiling heat transfer through. As a powerful cooling technology, boiling heat transfer performances regarding heat dissipation capacity (i.e., critical heat flux) and efficiency (i.e. heat transfer coefficient) can be enhanced through interfacial morphology and wettability manipulation with Graphene's intrinsic merits we already know. Carbon nanotube, which is in the same carbon allotrope with Graphene, was spotlighted as a potentate for boiling heat transfer once. However, it was not effective to clinch great success on boiling improvement due to its unfavorable characteristics such as hydrophobic nature preventing coolant refreshment and complex and dense coalescence of itself under twophase flow system. Even through there are few approaches about boiling heat transfer using functionalized Graphene manipulating wettability characteristics so far, many researchers have tried to find a way for controllable wettability on Graphene and its stability [9-11]. A few recent studies showed the possibility of Graphene on the application. During the boiling using Graphene-nanofluid it came to deposit on the heat-dissipating surface by thermophoretic effect accompanying heterogeneous vaporization [2]. By controlling operating conditions, Graphene could form boiling-favorable interfacial surface with secondary cavity-like structures on a heat transfer surface, and it would be effective for the vaporization of coolant. The consequent heat dissipation would be improved with extended heat-dissipating capacity and efficiency [12]. Besides those advantages, we can stress that Graphene has remarkable physical and thermal characteristics including young's modulus, yield strength and thermal conductivity $[13,14]$, thus we can expect long-term stability and robustness different superior to other nanomaterials.

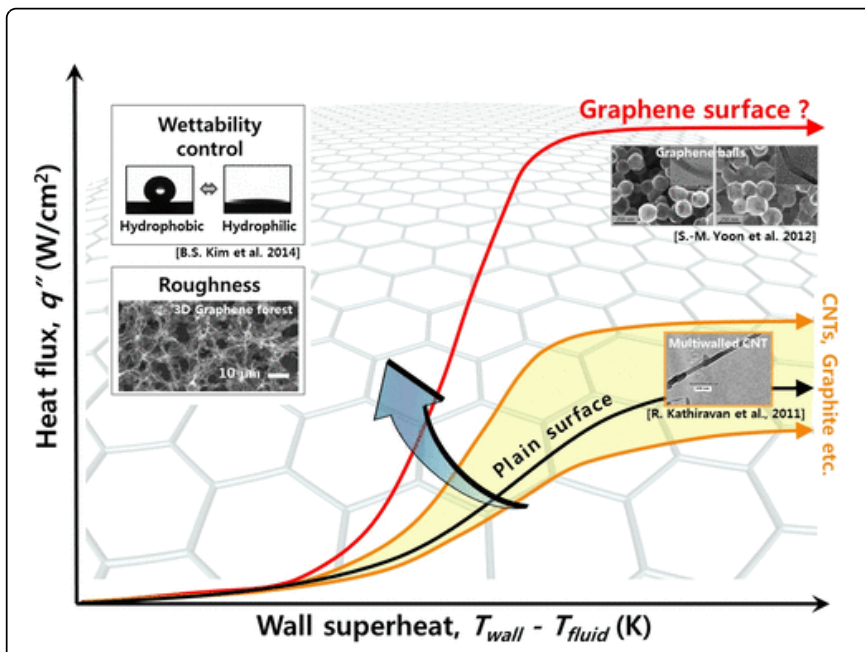

Figure 1: Surface roughness and wettability control are the principle factor to enhance the performance of boiling heat transfer. Graphene surface will lead to long-term stability and robustness boiling condition through outstanding physical/thermal properties.

The study on heat transfer using Graphene is just beginning step despite remarkable properties of Graphene, and more study is necessary to investigate and improve about Graphene's characteristics for heat transfer application: the boiling heat transfer mechanism on Graphene coated surface, wettability control for super hydrophilic and wicking characteristics. Furthermore, suitable structure for vaporization can be designed in 3-D geometry using thermophoresis, thermal-CVD and PE-CVD. Also, uniform and reliable coating method should be developed. Then Graphene will lead to the 
Citation: Cho HH, Choi G, Shim DI (2014) Can Graphene Lead to Breakthrough in Boiling Heat Transfer?. J Material Sci Eng 3: e109. doi:

Page 2 of 2

breakthrough for enormously improved heat transfer technology in industry fields.

\section{Acknowledgments}

This work was supported by a National Research Foundation of Korea (NRF) grant funded by the Korea government (MEST) (No. 2011-0017673) and the Human Resources Development program (No. 20134030200200) of the Korea Institute of Energy Technology Evaluation and Planning (KETEP) grant funded by the Korea government Ministry of Trade, Industry and Energy.

\section{References}

1. Balandin AA, Ghosh S, Bao W, Calizo I, Teweldebrhan D, et al. (2008) Superior Thermal Conductivity of Single-Layer Graphene. Nano Lett 8: 902-907.

2. Park SD, Lee SW, Kang S, Bang IC, Kim JH, et al. (2010) Effects of nanofluids containing grapheme/graphene-oxide nanosheets on critical heat flux. Appl Phys Lett 97: 023103.

3. Chen R, Lu MC, Srinivasan V, Wang Z, Cho HH, et al. (2009) Nanowires for enhanced boiling heat transfer. Nano Lett 9: 548-553.

4. Kandlikar SG (2013) Controlling bubble motion over heated surface through evaporation momentum force to enhance pool boiling heat transfer. Appl Phys Lett 102: 051611.

5. Kim BS, Shin S, Shin SJ, Kim KM, Cho HH (2011) Micro-nano hybrid structures with manipulated wettability using a two-step silicon etching on a large area. Nanoscale Res Lett 6: 333.
6. Kim BS, Shin S, Lee D, Choi G, Lee H, et al. (2014) Stable and uniform heat dissipation by nucleate-catalytic nanowires for boiling heat transfer. Int J Heat Mass Transf 70: 23-32.

7. Yoon SM, Choi WM, Baik H, Shin HJ, Song I, et al. (2012) Synthesis of Multilayer Graphene Balls by Carbon Segregation from Nickel Nanoparticles. ACS Nano 6: 6803-6811.

8. Kathiravan R, Kumar R, Gupta A, Chandra R, Jain PK (2011) Pool boiling characteristics of multiwalled carbon nanotube (CNT) based nanofluids over a flat plate heater. Int J Heat Mass Transf 54: 1289-1296.

9. Rafiee J, Rafiee MA, Yu ZZ, Koratkar N (2010) Superhydrophobic to Superhydrophilic Wetting Control in Graphene Films. Adv Mater 22: 2151-2154.

10. Rafiee J, Mi X, Gullapalli H, Thomas AV, Yavari F, et al. (2012) Wetting transparency of graphene. Nat Mater 11: 217-222.

11. Raj R, Maroo SC, Wang EN (2013) Wettability of graphene. Nano Lett 13: 1509-1515.

12. Ahn HS, Jang JW, Seol M, Kim JM, Yun DJ, et al. (2013) Self-assembled foam-like graphene networks formed through nucleate boiling. Sci Rep 3: 1396.

13. Jiang JW, Wang JS, Li B (2009) Young's modulus of graphene: a molecular dynamics study. Phys Rev B 80: 113405 .

14. Lee C, Wei X, Kysar JW, Hone J (2008) Measurement of the elastic properties and intrinsic strength of monolayer graphene. Science 321: 385-388. 